\title{
Restoring single-molecule localizations with wavefront sensing adaptive optics for deep-tissue super-resolution imaging
}

\author{
Sanghyeon Park ${ }^{1,2,+}$, Yonghyeon Jo ${ }^{1,2,+}$, Minsu Kang ${ }^{3}$, Jin Hee Hong ${ }^{1}$, Sangyoon $\mathrm{Ko}^{3}$, Suhyun Kim ${ }^{5}$, Sangjun \\ Park $^{4}$, Hae-Chul Park ${ }^{5}$, Sang-Hee Shim ${ }^{3, *}$, and Wonshik Choi ${ }^{1,2, *}$ \\ ${ }^{I}$ Center for Molecular Spectroscopy and Dynamics, Institute for Basic Science, Seoul 02841, Korea \\ ${ }^{2}$ Department of Physics, Korea University, Seoul 02855, Korea \\ ${ }^{3}$ Department of Chemistry, Korea University, Seoul 02855, Korea \\ ${ }^{4}$ Department of Medical Life Sciences, The Catholic University of Korea, Seoul 06591, Korea \\ ${ }^{5}$ Department of Biomedical Sciences, Korea University, Asan 425-707, Korea \\ ${ }^{+}$These authors contributed equally to this work \\ *sangheeshim@korea.ac.kr andwonshik@korea.ac.kr
}

\begin{abstract}
Specimen-induced aberration has been one of the major factors limiting the imaging depth in single-molecule localization microscopy (SMLM). In this study, we measured the wavefront of intrinsic reflectance signal at the fluorescence emission wavelength to construct a time-gated reflection matrix and find complex tissue aberration without resorting to fluorescence detection. Physically correcting the identified aberration via wavefront shaping with a liquid-crystal spatial light modulator (SLM) enables super-resolution imaging even when the aberration is too severe for initiating localization processes. We demonstrate the correction of complex tissue aberration, the root-mean-square (RMS) wavefront distortion of which is more than twice the $1 \mathrm{rad}$ limit presented in previous studies; this leads to the recovery of single molecules by 77 times increased localization number. We visualised dendritic spines in mouse brain tissues and early myelination processes in a whole zebrafish at up to $102 \mu \mathrm{m}$ depth with 28-39 nm localization precision. The proposed approach can expand the application range of SMLM to thick samples that cause the loss of localization points owing to severe aberration.
\end{abstract}

\section{Introduction}

Single-molecule localization microscopy (SMLM) improves the spatial resolution of a diffraction-limited fluorescence microscope by more than an order of magnitude ${ }^{1-3}$. The approach has widely been used in diverse biological studies owing to its simplicity and high resolution $(<30 \mathrm{~nm})^{4}$. However, its working depth is much shallower than those of typical deep-tissue imaging modalities such as confocal and multi-photon fluorescence microscopy. This is mainly because single-molecule localization (SML) is highly susceptible to reductions in the signal-to-noise ratio (SNR) and the distortion of the point spread function (PSF) induced by tissue scattering and aberration. More specifically, PSF fitting is limited owing to background fluorescence from molecules at defocused planes in a bulk tissue. Strategies that selectively excite the plane of interest have been proposed to reduce such background noise ${ }^{5,6}$. PSF blur due to tissue-induced aberration reduces the number of emitted photons detected by camera pixels at PSF peaks. This results in SNR reduction and loss of localizations ${ }^{7,8}$. In addition, the distortion of the PSF shape can result in erroneous localization and, hence, degrade localization precision.

Adaptive optics (AO) that actively control the PSF with wavefront shaping devices (such as a deformable mirror and a liquid-crystal spatial light modulator (SLM)) are suitable solutions for coping with tissue aberrations. The method was first introduced in astronomy to deal with atmospheric turbulence ${ }^{9}$ and adopted in optical microscopy to recover the diffraction-limited resolution in deep-tissue imaging ${ }^{10}$. In recent years, $\mathrm{AO}$ has been implemented in different super-resolution fluorescence microscopy methods including SMLM ${ }^{11,12}$, stimulated emission depletion (STED) microscopy ${ }^{13,14}$, and structured illumination microscopy (SIM) ${ }^{15}$. AO super-resolution imaging approaches can largely be categorised into two types. In the first approach, the wavefront of fluorescence emissions is directly measured from either artificial ${ }^{16}$ or intrinsic ${ }^{17}$ point sources within the sample with a ShackHartmann wavefront sensor. In this so-called wavefront sensing AO method, two-photon fluorescence emissions have often been used as guide stars without using fluorescent particles. However, this approach has not yet been implemented in SMLM. One can consider measuring the wavefront of single-molecule emissions as they can serve as guide stars, but their signals are too weak for the wavefront measurement. For this reason, the other type (i.e., sensorless AO) has widely been used in SMLM.

In sensorless AO, the wavefront shaping device is controlled such that the image quality metric of the SMLM images is optimised ${ }^{13,14,18}$. In all cases, a deformable mirror has been used to determine the specific amplitude of each Zernike mode that maximises the image metric. The image sharpness metric emphasising higher spatial frequency components of the reconstructed SMLM image has mainly been used owing to its insensitivity to intensity fluctuations. These approaches enable successful SMLM imaging across entire depth of cells and within tissues $^{11,12,19}$. However, recording SMLM images in each optimization step imposes several constraints. First, the aberration degree should be sufficiently mild for detecting single-molecule emissions above the background noise. 
Otherwise, evaluating the image metric and initiating the optimization process is impossible. Second, recording multiple images in each iteration step takes too much time, which results in the loss of fluorophores due to photobleaching. Third, the optimization process is typically nonlinear; its efficiency is highly dependent on the choice of the image quality metric and optimization method ${ }^{18}$. All these constraints preclude the correction of complicated high-order aberration, thereby limiting the achievable imaging depth in SMLM. In fact, most of the previously presented implementations can deal with mild aberrations, the root-mean-square (RMS) wavefront distortion of which is typically less than $1 \mathrm{rad}$. Therefore, the main benefit of the previously presented AO modalities is improving the image contrast rather than recovering unseen structures ${ }^{11-13}$. Even when the image quality seems improved, the imaging depth is still only a couple of tens of microns ${ }^{13}$. The computational approach that finds an aberrated PSF in situ via the phase retrieval of single-molecule emission PSFs is another useful form of sensorless $\mathrm{AO}^{20}$. However, its working depth is still limited owing to the lack of physical correction methods for aberration without wavefront shaping devices.

The label-free wavefront sensing AO method termed 'closed-loop accumulation of single scattering (CLASS) microscopy ${ }^{21,22}$ can be a suitable solution for overcoming the major limitations of previously presented AOSMLM methods. CLASS microscopy records multiple interferometric reflectance images (not fluorescence images) from intrinsic heterogeneous tissue structures at different illumination angles. Sample-induced aberration is identified based on the reflection matrix constructed from the set of measured reflectance images. Since computational post-processing is performed with a unique linear optimization algorithm, extremely complex tissue aberrations of even invisible structures underneath an intact mouse skull can be identified ${ }^{23}$. Essentially, CLASS microscopy measures complex tissue aberration from intrinsic reflectance images; the method does not rely on fluorescence detection. This enables its integration into SMLM without the loss of fluorescent molecules during aberration calculation. Furthermore, it can be used in extreme conditions in which fluorescent PSFs are initially invisible.

In this paper, we propose the integration of CLASS microscopy into SMLM. In this CLASS-SMLM approach, the wavelength of the light source for CLASS microscopy is tuned to the fluorescence emission band of SMLM. By displaying the phase conjugation of aberration identified with CLASS microscopy on a liquid-crystal SLM placed in the emission beam path of the SMLM setup, we corrected complex aberrations whose RMS wavefront distortion exceeded more than twice the 1 rad limit of previously presented modal-based approaches. Because this aberration measurement method does not rely on SMLM, it can be applied even when the aberration is too severe to initiate a localization process. We applied CLASS-SMLM for tissue imaging and performed super-resolution imaging of mouse brain tissues and a whole zebrafish at up to $102 \mu \mathrm{m}$ depth. The correction of sample-induced aberration recovered localization precision of $28-39 \mathrm{~nm}$ and increased the localization number by 77 times. These features enable the visualization of fine structures such as dendritic spine necks and nascent myelin sheaths generated during the initiation of myelination that are invisible without CLASS-SMLM.

\section{Results}

\section{CLASS-SMLM}

The deep-tissue super-resolution microscopy system was built on a commercial inverted microscope (Fig. Figure 1a). One port of the microscope was connected to a CLASS microscope (yellow box in Fig. Figure 1a), and the other was connected to the single-molecule localization (SML) microscope (cyan box in Fig. Figure 1a). They shared a sample, an objective lens, and a tube lens. One could switch from one microscope system to the other with a built-in flip mirror in the microscope (Methods and Supplementary Note). The CLASS microscope was equipped with a superluminal laser diode with a broad bandwidth. Its short coherence time (approximately 180 fs) allowed time-gated detection for rejecting unwanted reflections from out-of-focus planes. Its center wavelength $(\lambda=678 \mathrm{~nm})$ was near the emission peak wavelength of Alexa Fluor 647 used for SMLM. The CLASS microscope recorded multiple interference images of intrinsic elastic backscattered light from the sample at different illumination angles. Based on these images, sample-induced aberration was identified by applying an algorithm that maximises the single-scattering intensity (Supplementary Note).

The SMLM setup was equipped with an excitation source $(637 \mathrm{~nm})$ and an activation source $(405 \mathrm{~nm})$. The emitted beam (dotted lines in Fig. Figure 1a) captured by the objective lens (1.2 NA, water immersion lens) was split into two beam paths by a polarising beam splitter (PBS1 in Fig. Figure 1a). In the beam path of the horizontal polarization component, we installed an SLM at a plane conjugate to the pupil plane of the objective lens. To correct aberration, we displayed the opposite phase of the aberration map identified by the CLASS microscope. The size, position, and orientation of the aberration correction map displayed on the SLM were carefully calibrated to match the spatial coordinates of the CLASS microscope (Supplementary Note). The aberration-corrected emission beam arrived at one corner of the camera sensor (EMCCD) with $33 \times 33 \mu \mathrm{m}^{2}(256 \times 256$ pixels $)$ field of view. The other emission beam with vertical polarization was directly sent to another corner of the EMCCD sensor without passing through the SLM. This enabled the simultaneous recording of the aberration-uncorrected 
images (AO off in Fig. Figure 1a) and aberration-corrected images (AO on in Fig. Figure 1a). Before correcting the sample-induced aberration, the system aberration in the SMLM beam path was corrected with fluorescent beads as guide stars (Supplementary Note). The correction of system aberration reduced the PSF FWHM from $(\Delta x, \Delta y)=(437 \mathrm{~nm}, 474 \mathrm{~nm})$ to $(385 \mathrm{~nm}, 419 \mathrm{~nm})$ (Supplementary Figure SN5), which is slightly greater than the diffraction limit $(341 \mathrm{~nm}$ ) due to residual system aberration. As presented in the following paragraph, CLASSSMLM enables the correction of sample-induced aberrations and reduces the PSF width limited by diffraction and residual system aberration. The method improves the localization precision to $28-39 \mathrm{~nm}$ in various tissue samples (Supplementary Table 1); the improvement is sufficient for visualising the fine structures investigated in this study.

\section{Proof-of-concept of CLASS-SMLM for imaging microtubules in a COS-7 cell}

To assess the performance of the proposed CLASS-SMLM system, we imaged microtubules in a COS-7 cell immunolabelled with Alexa Fluor 647. The cells were cultured on a cover glass onto which 100 nm diameter gold nanoparticles had been attached. An aberration layer was inserted between the objective lens and cover glass. Its aberration degree was measured via interferometric reflectance imaging of the gold nanoparticles with CLASS microscopy (inset in Fig. Figure $1 \mathrm{f}$ with tilt and removed defocus Zernike modes). As demonstrated in the following paragraphs, there is no need to inject gold nanoparticles for tissue imaging (mouse brain tissues in Fig. Figure $\mathbf{2}$ and zebrafish embryos in Figs. Figure $\mathbf{3}$ and Figure 4) because CLASS microscopy exploits the intrinsic reflection signals from inhomogeneous tissue structures to identify aberration at any depth of interest.

The effect of the corrected aberration on the single-molecule PSFs is evident when the representative raw images without (Fig. Figure 1b) and with (Fig. Figure 1c) AO recorded in the same camera frame are compared(Supplementary Video 1). Without AO, the PSF shapes were highly asymmetric and broadened, which made the single-molecule images barely visible above background noise level (Fig. Figure 1b). They were converted into sharp bright spots with AO (Fig. Figure 1c). The difference becomes clearer when the PSFs obtained based on the ensemble average of the single-molecule emission PSFs in the insets of Figs. Figure $\mathbf{1 b}$ and $\mathrm{c}$ are compared. In fact, the aberrated PSF in the inset of Fig. Figure $\mathbf{1 b}$ from single-molecule images was almost identical to that estimated from sample-induced aberration. Their intensity correlation was 0.91 , which proves the precise aberration measurement of CLASS microscopy. With AO, the FWHM of the ensemble-averaged PSF decreased from 1,425 to $380 \mathrm{~nm}$, which is identical to the PSF width of the residual system aberration. This indicates that all specimen-induced aberration was successfully removed. In addition, the Strehl ratio (i.e., the aberrated PSF peak intensity-to-ideal PSF peak intensity ratio) was improved by 4.3 times. All these features confirm the precise aberration correction ability of CLASS-SMLM.

The aberration degree in Fig. Figure If was far higher than that addressable by the previously presented modalbased optimization approaches ${ }^{12,13}$. The RMS wavefront distortion of the aberration map (inset in Fig. Figure 1f) was $2.2 \mathrm{rad}$, which is more than 2 times greater than the $1 \mathrm{rad}$ limit of the other approaches. This aberration level requires the correction of, at least, the first 100 Zernike modes (Supplementary Fig. 1), which is far beyond the capacity reached in previous AO studies (the researchers have mostly dealt with the first 20 Zernike modes). The use of a liquid-crystal SLM rather than a deformable mirror and the precise aberration measurement with the CLASS microscope made aberration correction at this high aberration level possible.

From a series of single-molecule emission PSFs including those in Figs. Figure $\mathbf{1 b}$ and c, we reconstructed SMLM images without (Fig. Figure 1f) and with (Fig. Figure 1g) AO with the ThunderSTORM software (see Methods $)^{24}$. With AO, the image resolution was significantly improved, which resulted in clearly resolved microtubules. The quality of the diffraction-limited fluorescence images was similarly improved (Figs. Figure 1d and e). However, many overlapping microtubules were only resolved in the AO-on SMLM image.

The detailed analysis indicates that the improved localization precision and localization number enhanced the image quality (Supplementary Fig. 2). According to the nearest neighbor analysis results presented below, localization precision was improved by 2.6-3.6 times. Furthermore, the AO increased the localization number by 14-25 times, which is remarkable compared with those of the previously published AO-SMLM studies (their localization numbers increased by approximately $2-5$ times $^{12,18}$ ). This confirms that our proposed system can correct severe aberration.

For further analysis, we selected two representative regions of interest: one with a single isolated microtubule (yellow box in Figs. Figure $\mathbf{1} \mathrm{f}$ and $\mathrm{g}$ ) and the other with densely populated microtubules (green box in Figs. Figure 1f and g). Their zoom-in images are presented in Figs. Figure 1h-k. In the yellow box, it was difficult to even pinpoint the single microtubule without AO (Fig. Figure 1h), whereas it was sharply resolved with AO (Fig. Figure 1i). Its FWHM was measured (69 nm; Fig. Figure 11) by analysing the cross-sectional profiles in the white boxes in Figs. Figure $\mathbf{1 h}$ and i. The measured value is reasonable considering the known microtubule diameter (approximately $25 \mathrm{~nm}$ ) and size of primary and secondary antibodies (10-15 nm). It also agrees well with the values presented in previous reports ${ }^{25,26}$. It should be noted that the cross-sectional profile without AO was hardly 
visible compared to that with AO (Fig. Figure 11) owing to the significantly decreased localization number. The effect of AO was also pronounced in the green box. The cross-sectional profiles (Fig. Figure 1n) of the white boxes in Figs. Figure $\mathbf{1 j}$ and $\mathrm{k}$ show that two microtubules separated by $173 \mathrm{~nm}$ were invisible without AO; however, they could be clearly resolved with AO. With AO, the measured FWHM values of the microtubules were 82 and $85 \mathrm{~nm}$, respectively.

We estimated the lateral localization precision by a nearest neighbor analysis ${ }^{27}$. Figs. Figure $1 \mathrm{~m}$ and o show the probability density distributions of the distances among single-molecule PSFs. They were fitted with a composite function composed of a non-Gaussian term describing the contribution of individual single molecules and a Gaussian term describing the contribution of neighboring single molecules (see Methods). Without AO, both the Gaussian and non-Gaussian terms made significant contributions to the distribution (red curves in Figs. Figure $1 \mathrm{~m}$ and o). We discovered that the contribution from neighboring molecules was more pronounced in the densely populated regions without AO owing to overlapping broadened PSFs (Supplementary Fig. 3). With AO, the nonGaussian term contributed exclusively to the probability density distributions (blue curves in Figs. Figure $1 \mathrm{~m}$ and o). This suggests that the CLASS-SMLM method attenuated the effect of neighboring molecules in the localization process. The lateral localization precision estimated with the non-Gaussian terms for the yellow and green boxes was 57 and $83 \mathrm{~nm}$ without AO, respectively; these values decreased to 22 and $23 \mathrm{~nm}$ with AO, respectively. This improvement can largely be attributed to the reduction in the PSF width by a factor of 0.27 along with the reduced contribution of neighboring molecules. Furthermore, we conducted Fourier ring correlation (FRC) analysis to verify the combined effect of the localization precision and localization number density ${ }^{28}$. The FRC analysis results revealed the clear broadening of the spatial frequency bandwidth and the improvement of the spatial resolution from 134 to $41 \mathrm{~nm}$ (Supplementary Fig. 4).

\section{Deep-tissue SMLM imaging in mouse brain tissues}

To use more realistic samples than a cell with an aberration layer, approximately $150 \mu \mathrm{m}$ thick brain tissue slices of Thy1-EGFP transgenic mice were selected. We targeted dendritic spines of neurons expressing GFP that have been labelled with anti-GFP antibodies conjugated with Alexa Fluor 647 (see Methods). We collected inherent reflectance images from the biological structures such as cell bodies, blood vessels, and myelins at $50 \mu \mathrm{m}$ depth (Supplementary Fig. 5) and obtained a tissue aberration map (bottom-left inset in Fig. Figure 2a) via CLASS microscopy. We have never used guide stars such as gold nanoparticles when imaging brain tissues or an intact zebrafish. The identified sample aberration corresponds to $1.4 \mathrm{rad}$ RMS wavefront distortion, which is beyond the 1 rad limit of previously presented AO-SMLM methods.

In the next step, we performed SMLM imaging of the neuronal processes (Figs. Figure 2a-c). By comparing the AO-off and -on ensemble-averaged PSFs, we verified that the AO had reduced the PSF width by a factor of 0.62 with a Strehl ratio increase of 2.8 (Supplementary Table 1). This PSF improvement was less than that achieved for the cell with an aberration layer since the aberration degree was less severe (RMS values of $1.4 \mathrm{rad}$ in the tissue and $2.2 \mathrm{rad}$ in the cell with an aberration layer). Consequently, the difference between the AO-off and -on diffraction-limited fluorescence images was less pronounced (top-right insets, Fig. Figure 2a). By contrast, the SMLM images reveal a substantial difference between the AO-off and -on results (Fig. Figure 2a). Although thick stems of neural structures were somewhat visible even without AO, the heads and fine necks of dendritic spines were only properly reconstructed in the AO-on SMLM image (Figs. Figure $2 \mathrm{~b}$ and c). In addition, the difference between the localization precisions of both SMLM images was almost twofold ( $\sigma_{\mathrm{AO} \text { off }}=66 \mathrm{~nm}$ and $\sigma_{\mathrm{AO} \text { on }}=38 \mathrm{~nm}$, Fig. Figure 2e). The difference between the diffraction-limited images and SMLM images can mainly be explained based on the number of localization points. Despite relatively mild aberration, the localization number was reduced by $90 \%$ without AO (Fig. Figure 2d). This is possibly due to background fluorescence from bulk tissues, which was 1.6 times higher than in the cell experiment (Supplementary Fig. 6). The decrease in the localization number reduced the point-limited resolution, often referred to as 'Nyquist resolution'. In the entire area, the mean distance between localization points was $9.7 \mathrm{~nm}$ for AO-off and $3.2 \mathrm{~nm}$ for AO-on images; this indicates an approximately three-fold difference in the Nyquist resolution. This explains why the SMLM image without AO failed to reconstruct fine structures (AO-off image in Fig. Figure 2a) although the diffraction-limited fluorescence image was only mildly blurred (top-right insets in Fig. Figure 2a). Another image set of mouse brain tissue shows a similar tendency (Supplementary Fig. 7), which supports this explanation.

To see the effect of the imaging depth on the CLASS-SMLM performance, we applied our method to a deeper depth $(74 \mu \mathrm{m})$ for similarly prepared, approximately $200 \mu \mathrm{m}$ thick mouse brain tissue (Figs. Figure $\mathbf{2 f}-\mathrm{k}$ ). It is noteworthy that $74 \mu \mathrm{m}$ imaging depth is the deepest depth in mouse brain tissues reported so far. We discovered that many parameters at this depth were close to those at $50 \mu \mathrm{m}$ depth. The aberration correction map (top-left inset in Fig. Figure 2f) looks similar to that at $50 \mu \mathrm{m}$ depth. In addition, the effects of AO on the ensembleaveraged PSFs were similar. With AO, the ensemble-averaged PSF width was reduced by 0.54 times, and the Strehl ratio increased by 3.9 times. The SMLM images without and with AO were also qualitatively similar (Figs. 
Figure $2 \mathrm{~g}-\mathrm{i}$ ). At $74 \mu \mathrm{m}$ depth, the heads and fine necks of dendritic spines were clearly resolved only with AO, such as in the case at $50 \mu \mathrm{m}$ depth. The localization precision $\left(\sigma_{\mathrm{AO} \text { off }}=60 \mathrm{~nm}\right.$ and $\sigma_{\mathrm{AO} \text { on }}=37 \mathrm{~nm}$, Fig. Figure $2 \mathrm{k}$ ) at both depths were similar as well. However, the photon numbers were significantly different. The mean photon number per emission PSF at $74 \mu \mathrm{m}$ depth (960 with AO in Fig. Figure 2j) was 0.46 times that at $50 \mu \mathrm{m}$ depth (2,100 with AO in Fig. Figure 2d), thereby suggesting that the photon collection efficiency was reduced owing to the depth and sample-induced scattering. Interestingly, the background fluorescence noise level was higher at $50 \mu \mathrm{m}$ depth, which is probably due to the bright structures near the imaging view field (Supplementary Figure 6). This strongly fluorescent background seems to be responsible for more reduction of localization number at $50 \mu \mathrm{m}$ depth than at $74 \mu \mathrm{m}$ depth.

\section{Deep-tissue SMLM imaging in a whole zebrafish}

As demonstrated in Fig. Figure 1, CLASS-SMLM can correct a higher degree of aberration than that of mouse brain tissues. Therefore, we applied the method to whole zebrafish embryos whose complex and heterogeneous skin architectures introduce pronounced aberrations ${ }^{22}$. For SMLM imaging, an intact 3.5 days post-fertilization (dpf) Tg(claudinK:gal4vp16; uas:megfp) zebrafish with GFP on the plasma membrane of oligodendrocyte ${ }^{29}$ was $^{2}$ fixed and immunolabelled with anti-GFP antibodies conjugated with Alexa Fluor 647. The fish was mounted with its back against the coverslip (Fig. Figure 3a). Its head and tip of the tail were attached to the cover glass with a UV glue. The coverslip with the glued fish was inserted into a magnetic imaging chamber and immersed in imaging buffer with $\beta$-mercaptoethanol as switching agent and an oxygen scavenging system based on glucose oxidase (see Methods). The age of the fish was chosen such that early myelination could be captured in which oligodendrocytes start to cover the neuronal axons with nascent myelin sheaths ${ }^{30,31}$. The spinal cord of the fish near the trunk was chosen to resolve structures in early myelination in the central nervous system (Fig. Figure 3b). At $52 \mu \mathrm{m}$ depth from the coverslip, we found myelinating oligodendrocytes forming nascent myelin sheaths around axons along the spine ( $y$ axis), as shown in Fig. Figure $\mathbf{3} \mathrm{c}^{32}$. The aberration correction map in this area (inset in Fig. Figure 3d) was more complex than those of mouse brain tissues (insets in Figs. Figure 2a and f). The RMS wavefront distortion (1.98 rad) was approximately 1.5-2 times larger than those of the brain tissues. Its Strehl ratio increase was 3.6. Owing to image distortion due to this aberration, the conventional fluorescence image without AO (top, Fig. Figure 3c) had less intensity and no fine features in comparison with the AO-on image (bottom, Fig. Figure 3c).

The CLASS-SMLM approach corrected these distortions and recovered many structures, which were invisible without AO (Fig. Figure 3d). In particular, the fine necks and feet of nascent sheaths were well resolved with AO, whereas they could not be reconstructed without AO (Figs. Figure $3 \mathrm{e}$ and $\mathrm{f}$ ). It should be noted that the AO-on SMLM image resolved a higher density of localization points at the sole of the myelin sheath head (Fig. Figure 3e), which may indicate the initial wrapping of the axon by the oligodendrocyte membrane ${ }^{33,34}$. Moreover, the double-peak cross-sectional profile was well resolved with AO, whereas it was blurred into a single-peak profile without AO (Fig. Figure 3g). This substantial difference between the AO-off and -on SMLM images was mostly due to the different localization numbers (Fig. Figure $3 \mathrm{~h}$ ). In the entire image area, the localization number of the AO-off SMLM image was only $9.2 \%$ of that of the AO-on SMLM image. Interestingly, the difference between AO-off and -on SMLM images varied depending on the location. This was because the reduction degree of the localization number varied spatially. For example, they were $4.7 \%, 1.3 \%$, and $5.7 \%$ for the areas shown in Figs. Figure $3 \mathrm{e}-\mathrm{g}$. This decrease in the localization number translates into a reduced point-limited resolution. In the AO-off SMLM image, the mean distances between localization points were 21, 44, and $16 \mathrm{~nm}$ for Figs. Figure $3 \mathrm{e}-\mathrm{g}$, respectively. Considering that several-fold oversampling relative to the Nyquist criterion is recommended for SMLM, the degradation of the localization density was responsible for the loss of fine features without AO. By contrast, the mean distance between localization points decreased to 4.5, 5.0, and $3.8 \mathrm{~nm}$ with AO in Figs. Figure $3 \mathrm{e}-\mathrm{g}$, respectively, which provided sufficient localization density for resolving the myelin sheaths. The improved localization precision and mean distance between localization points affected the final SMLM sampling quality. This was evident in the Fourier ring correlation analysis in which the distinct broadening of the spatial frequency bandwidth was visible with AO (Supplementary Fig. 4).

To investigate the performance at a deeper depth and higher degree of aberration, we imaged a more developed, 5-dpf zebrafish embryo (Fig. Figure 4). At $102 \mu \mathrm{m}$ depth, the field of view exhibited severe aberration with RMS wavefront distortion of $2.64 \mathrm{rad}$. Remarkably, this degree of aberration exceeds that of the cell with the artificial aberration layer in Fig. Figure 1. The steep curvature of the phase map (top-right inset in Fig. Figure 4a) yielded a severely elongated PSF along the spinal cord (bottom-left inset in Fig. Figure 4a). This significantly decreased the number of localizations, thereby making the SMLM image nearly unreadable (Fig. Figure 4a). By contrast, CLASS-SMLM retrieved a sharp PSF (bottom-left inset in Fig. Figure 4b) by reducing its width by 4.1 times (Supplementary Table 1 and Supplementary Video 2). The corrected PSF resulted in $\sigma_{\mathrm{AO} \text { on }}=34$ nm localization precision, which is on a par with the other localization precision results obtained at shallower depths 
(Supplementary Table 1). However, without AO, the localization precision could not be determined in the nearest neighbor analysis because of the small number of localization points (Fig. Figure 4e). In fact, CLASS-SMLM collected 16.2 times more localization points (Fig. Figure 4e), which enabled the reconstruction of fine tubular structures with FWHMs of 150-160 nm (Fig. Figure 4b). The diffraction-limited (Fig. 4d) and SMLM images with AO revealed uneven fluorescence intensities along the axons. This indicates that the oligodendrocytes were unevenly wrapping axons ${ }^{33,34}$. It should be noted that axons in the conventional fluorescence image without AO (Fig. Figure 4c) appeared as smooth lines owing to the elongated PSF along the spinal cord direction. This may give the false impression that these axons were continuous and uniform. It is noteworthy that the degree of aberration varied drastically depending on the location within the sample. In another example (Supplementary Figure 8) at a similar depth $(100 \mu \mathrm{m})$, a much lower degree of aberration (RMS of 1.14) was observed with a mild loss of localization points (4.1 times). Our CLASS-SMLM approach could recover a similar image quality level regardless of the degree of aberration.

\section{Discussion}

We introduced an AO super-resolution imaging modality termed 'CLASS-SMLM' for investigating highly aberrated specimens such as tissue slices and whole organisms. CLASS microscopy was employed as a label-free wavefront sensing AO method to determine tissue aberration based on intrinsic reflection signals. Moreover, an aberration correction map was applied to an SLM in the fluorescence emission path of the SMLM setup to reconstruct super-resolved fluorescence images. Our method increases the degree of correctable aberration by more than twice in terms of the RMS wavefront distortion compared with previously presented fluorescencebased AO-SMLM methods. The method is particularly well suited for correcting the complex degree of aberration because of the unique linear optimization algorithm and the physical correction of aberration with a liquid-crystal SLM. Hence, CLASS-SMLM can restore emission PSFs lost by tissue aberration, thereby increasing the number of localization points by up to 77 times (Supplementary Table 1). In addition, the localization precision is improved from 38-83 nm to 22-39 nm at $102 \mu \mathrm{m}$ depth. Essentially, CLASS-SMLM corrects unexplored degrees of aberration and achieves a high SMLM image quality similar to those of samples with little aberration. This enables deep-tissue imaging of mouse brain tissues and whole-animal imaging of zebrafish embryos.

In highly aberrated samples with aberration degrees beyond those previously explored, the effect of aberration on SMLM was much more severe and complicated than in diffraction-limited fluorescence microscopy. The aberration-distorted PSF and its peak intensity were lowered, its width was broadened, and its shape deviated from a symmetric Gaussian function. After the combination with comparable levels of noise and background, these distortions in the extremely weak signals of single fluorophores led to the loss of localization points in multiple stages of the ThunderSTORM analysis. When the peak intensity became comparable to or lower than the background level, the single-molecule emission PSFs became undetectable by the peak finding algorithm. Furthermore, during the fitting step for measuring the centroid positions, the distortion of PSF shapes such as broadening can lead to high fitting errors and, hence, erroneous localizations. This degraded the localization precision of isolated fluorophores, in particular, when the PSFs of fluorophores in proximity overlapped. The direct comparison of uncorrected and corrected SMLM images reconstructed from identical emission PSFs suggests that the major issue caused by tissue aberration is the substantial loss of localization points (up to 98.7\%). In addition, the localization precision was degraded, yet the difference was relatively small (up to 3.6 fold deviation). Therefore, the PSF width and shape were less of an issue than the PSF height. This was also observed in the mouse brain tissues at different depths (Figs. Figure $\mathbf{2 a}$ and $\mathrm{f}$ ). Figure Figure $\mathbf{2} \mathrm{f}$ was obtained at a deeper depth $(74 \mu \mathrm{m})$ than Fig. Figure $\mathbf{2 a}(50 \mu \mathrm{m})$. The average photon number was smaller at the deeper depth owing to scattering. However, the loss of localization points at the shallower depth was approximately twice more severe than that at the deeper depth because the background fluorescence level was higher at the shallower depth (Supplementary Fig. 6). Therefore, we concluded that the degradation of the signal-to-background ratio of emission PSFs and resultant loss of localization numbers were the major hurdles in SMLM imaging in deep highly aberrated samples. We also observed that the effect of aberration varied locally in the field of view (Supplementary Fig. 4). This can also be attributed to the spatial inhomogeneity of the background noise. Nevertheless, the recovery of spatial resolution was fairly uniform with AO.

The localization precision obtained with CLASS-SMLM was on a par with the one of aberration-free cell samples $^{26,35}$. However, the absolute localization precision was slightly worse than that of commercial SMLM microscopes. Many factors contributed to the degraded localization precision: (1) A water immersion objective with NA 1.2 was used for the long working distance instead of oil objectives with NA > 1.4, which are usually used for SMLM. The NA 1.2 objective was selected for future research studies of SMLM imaging beyond 200 $\mu \mathrm{m}$ depth; (2) The photon budgets were divided into two beam paths for simultaneously collecting both correctionfree and corrected SMLM images; (3) The incomplete correction of system aberration: the microscope system was remodelled to accommodate the CLASS microscope, which resulted in relatively high degree of system 
aberration. Although the system aberration was corrected, it was not completely removed. As a result, the PSF FWHM $(380 \mathrm{~nm})$ of the microscope system was slightly worse than the diffraction limit (341 nm). Factors (1) and (2) can easily be improved by upgrading the objective to one with a higher NA with a working distance of approximately $150 \mu \mathrm{m}$ and by incorporating another SLM in the correction-free path, respectively. Residual system aberration requires a more advanced correction method than the current common practices ${ }^{18}$.

CLASS identifies aberration without using fluorescence. That is, photobleaching does not occur during the aberration calculation. Therefore, CLASS-SMLM has a crucial advantage when the labelling efficiency is low or multicolor SMLM is required. For example, the previously presented approach that uses two-photon fluorescence spots as nonlinear guide stars requires additional labelling and, thus, the allocation of additional fluorescence channels for guide stars ${ }^{15}$. This may not be easy in multicolor SMLM. For example, several different emission wavelengths are used for SMLM imaging of synapse ${ }^{36,37}$. In this case, adding an additional channel may be expensive or impossible. Of course, single-molecule images of SMLM fluorophores can be used for finding aberrations. However, due to the weak intensities, a significant portion of single-molecule emission PSFs must be used for aberration estimation, which reduces the density-limited resolution of the resultant SMLM image.

Although the proposed CLASS-SMLM can correct aberration-induced loss of fluorescence signals, it still faces challenges especially in background suppression. The presented study was performed with an epi-fluorescence microscope, and the tissue samples were sparsely labelled to minimise background fluorescence. To address background noise, the microscope can be combined with selective illumination methods ${ }^{5,6}$ or the sample can be treated with tissue clearing protocols ${ }^{38}$. Since the CLASS beam path is independent of the excitation/emission paths in the SMLM system, CLASS-SMLM can be combined with two-photon activation ${ }^{15}$, obliqueillumination/detection schemes ${ }^{5}$, or selective plane illumination ${ }^{6}$. Currently, the application of CLASS-SMLM in in vivo imaging is limited by the collection speed of reflectance images at different angles and the time required for running the CLASS algorithm. We anticipate that CLASS-SMLM will be used as a workhorse in in vivo superresolution imaging with the development of fast CLASS methods and the reduction of background fluorescence.

\section{Methods}

CLASS microscope setup. A low-coherence 678 nm laser (SLD-261-HP2-DBUT-PM-PD-FC/APC, Superlum, coherence length: approximately $40 \mu \mathrm{m}$ ) was used to illuminate the samples for time gating. The beam was steered by a two-axis galvo mirror $(6210 \mathrm{H}$, Cambridge Technology) to scan the illumination angle. It was then divided into the sample beam (SB) and reference beam (RB). The RB passed through a diffraction grating (Ronchi 120 $\mathrm{lp} / \mathrm{mm}$, Edmund Optics) for off-axis interference imaging. Only the first-order diffraction of the RB was combined with the SB reflected from the sample at the beam splitter in front of the sCMOS camera (pco.edge $4.2 \mathrm{~m}, \mathrm{PCO}$ ). We recorded interference images while scanning the illumination angles in such a way to cover the entire numerical aperture range of the objective lens. The imaging depth was selected according to the objective lens focus and the position of a reference mirror mounted on a motorised actuator (Z825B, Thorlabs). The complete CLASS setup was built on a commercial inverted microscope (Eclipse Ti2-E, Nikon) equipped with a $60 \times / 1.2$ NA water immersion objective lens (UPLSAPO 60XW, Olympus). In addition, a reflection matrix was constructed based on the measured complex field maps, and the CLASS algorithm was applied to obtain the sample-induced aberrations (Supplementary Note for detailed process).

SMLM setup. GFP or Alexa Fluor 647 were excited with a $488 \mathrm{~nm}$ laser (OBIS 488-60 LS, Coherent) or a 637 nm laser (LP637-SF70, Thorlabs). A $405 \mathrm{~nm}$ laser (405 nm LX $50 \mathrm{~mW}$ laser system, OBIS) was used for activation of Alexa Fluor 647. These lasers were coupled and focused onto the objective back aperture for epiillumination. A $505 \mathrm{~nm}$ LED (M505L3, Thorlabs) was installed for low-magnification transmission imaging; it was used to select the region of interest. The emitted fluorescence from the sample was magnified by a factor of 2 to achieve a pupil diameter of $9.5 \mathrm{~mm}$ at the SLM (X13138-06, Hamamatsu). A set of $4 \mathrm{f}$ relays was designed for the final magnification (100), which yielded a $130 \mathrm{~nm}$ effective pixel size at the EMCCD camera (DU-888U3CS0-\#BV, Andor). In addition, emission filters (ET700/75 m and ET525/50 m, Chroma) were placed in front of the EMCCD camera to filter out unwanted signals. The SMLM setup and CLASS microscope shared the objective and tube lenses (Supplementary Note for full experimental setup).

SMLM analysis. All raw images of the blinking single-molecule emission PSFs were processed with the ThunderSTORM ${ }^{24}$ ImageJ Fiji ${ }^{39}$ plugin. The localization precision values of the obtained SMLM images were calculated using the nearest neighbor analysis ${ }^{27}$. It was assumed that a localised position $\mathbf{r}_{i}\left(t_{i}\right)$ in a frame at time $t_{i}$ has a nearest neighbor localised position $\mathbf{r}_{i, \mathrm{NN}}\left(t_{i+1}\right)$ in the next frame at time $t_{i+1}$. The pairwise displacement $d_{i}$ is defined as the displacement $d_{i} \equiv \mathbf{r}_{i}\left(t_{i}\right)-\mathbf{r}_{i, \mathrm{NN}}\left(t_{i+1}\right)$ between nearest neighbors. Once collected, the set of pairwise displacements $d_{i}$ can be presented in the form of a histogram. Subsequently, the envelope of this histogram is fitted with a Gaussian function with a non-Gaussian correction term. The correction term is required 
bioRxiv preprint doi: https://doi.org/10.1101/2021.11.18.469175; this version posted November 20, 2021. The copyright holder for this preprint (which was not certified by peer review) is the author/funder. All rights reserved. No reuse allowed without permission.

because some nearest neighbors may result from different molecules in close proximity rather than from identical molecules. Therefore, the fitting curve is expressed as follows:

$$
p(d)=A_{1} \frac{d}{2 \sigma^{2}} \exp \left[-\frac{d^{2}}{4 \sigma^{2}}\right]+A_{2} \frac{1}{\left(2 \pi \omega^{2}\right)^{1 / 2}} \exp \left[-\frac{\left(d-d_{\mathrm{C}}\right)^{2}}{2 \omega^{2}}\right]
$$

where $d$ is the pairwise displacement, $\sigma$ the Gaussian standard deviation defining the localization precision, and $\omega$ the Gaussian standard deviation of the correction term centered at $d_{\mathrm{C}}$.

Preparation of nanoparticle-coated cover glasses. $18 \times 18 \mathrm{~mm}^{2}$ cover glasses $(0101030$, Marienfeld) were sonicated in $1 \mathrm{M} \mathrm{KOH}$ solution (6592-3705, Daejung) for $30 \mathrm{~min}$. In the next step, they were washed with Milli$\mathrm{Q}$ water (Direct 8, Merck) to remove remaining KOH solution. When necessary, the sonicated cover glasses were coated with gold nanoparticles. In this case, the cover glasses were previously coated with poly-L-lysine (P8920100ML, Sigma Life Science) for $5 \mathrm{~min}$. Subsequently, $5 \times$ diluted $100 \mathrm{~nm}$ diameter gold nanoparticles (753688$25 \mathrm{ML}$, Sigma-Aldrich) were dropped onto the cover glasses. They were dried in an oven at approximately $60{ }^{\circ} \mathrm{C}$ overnight.

Immunolabelling of microtubules in COS-7 cells. The COS-7 cells were seeded on gold-nanoparticle-coated cover glasses and cultured overnight in DMEM (11965-092, Gibco) containing 10\% FBS (10082147, ThermoFisher) and 1\% penicillin-streptomycin (15070063, Thermo Fisher). Prior to fixation, the cells were washed three times with pre-warmed PBS $\left(37{ }^{\circ} \mathrm{C} ; 21-040-\mathrm{CV}\right.$, Corning) and treated with the pre-warmed extraction buffer $\left(37{ }^{\circ} \mathrm{C} ; 0.125 \%\right.$ Triton X-100 (X100-500ML, Sigma-Aldrich) and $0.4 \%$ glutaraldehyde (G752610ML, Sigma-Aldrich) in PBS). Immediately after the treatment, the extraction buffer was quickly aspirated and rinsed three times with PBS. In the next step, the cells were fixed with the pre-warmed fixation buffer $\left(37^{\circ} \mathrm{C}\right.$; $3.2 \%$ paraformaldehyde (PC2031-100-00, Biosesang) and 0.1\% glutaraldehyde in PBS for 10 min at room temperature (RT). After fixation, they were treated with $10 \mathrm{mM}$ fresh sodium borohydride in PBS for $5 \mathrm{~min}$ at RT while being shaken. After three washing cycles with PBS, the cells were permeabilised with 3\% BSA (A703050G, Sigma Life Science) and 0.5\% Triton X-100 in PBS. In the next step, the primary antibody (ab6046, Abcam) 1000 times diluted in the blocking buffer (3\% BSA and 0.5\% Triton X-100 in PBS) was treated for $1 \mathrm{~h}$ at RT while the well plate was being shaken. The cells were then washed three times with PBS and treated with the secondary antibody (A-21245, Thermo Fisher), which was 1,000 times diluted in the blocking buffer for $1 \mathrm{~h}$ at RT while being shaken. After immunolabelling, the cells were washed three times with PBS and stored at $4{ }^{\circ} \mathrm{C}$ before the experiment.

Preparation of mouse brain slices. All experimental procedures were approved by the Committee of Animal Research Policy of Korea University (approval number KUIACUC-2019-24). Adult (over eight weeks old) Thy1EGFP line M (Jackson Labs \#007788) mice were deeply anesthetised with an intraperitoneal injection of 100 $\mathrm{mg} / \mathrm{kg}$ ketamine and $10 \mathrm{mg} / \mathrm{kg}$ xylazine. After decapitation, their brains were quickly excised and dropped into an ice-cold artificial cerebrospinal fluid (ACSF). The brains were cut into 150-200 $\mu \mathrm{m}$ thick coronal slices with a vibroslicer (World Precision Instruments, Sarasota, FL, USA) and fixed at $4{ }^{\circ} \mathrm{C}$ in $4 \%$ paraformaldehyde overnight before immunolabelling.

Preparation of zebrafish. The $\mathrm{Tg}$ (claudinK:gal4vp16;uas:megfp) zebrafish embryos were raised at $28{ }^{\circ} \mathrm{C}$ in $\mathrm{E} 3$ embryo medium ( $5 \mathrm{mM} \mathrm{NaCl}, 0.17 \mathrm{mM} \mathrm{KCl}, 0.33 \mathrm{mM} \mathrm{CaCl}_{2}$, and $0.33 \mathrm{mM} \mathrm{MgSO}_{4}$ ). After they had hatched, they were transferred to E3 medium containing N-phenylthiourea (Sigma) to inhibit pigmentation. After three to five days, the embryos were anesthetised with tricaine (Sigma) in E3 medium and fixed in $4 \%$ paraformaldehyde for $1-2 \mathrm{~h}$ at RT.

Immunolabelling of mouse brain slices and zebrafish. The fixed brain slices and intact zebrafish embryos were permeabilised for $3 \mathrm{~h}$ with a blocking buffer (3\% BSA and $0.5 \%$ Triton X-100 in PBS). Subsequently, the slices were immunolabelled for five days with $200 \times$ diluted anti-GFP Alexa 647-conjugated primary antibodies (A31852, Thermo Fisher) in the blocking buffer.

\section{Acknowledgements}

This work has been financially supported by the Institute for Basic Science (IBS-R023-D1) and the National Research Foundation (NRF) grants funded by the Korean government (MSIT) (2021R1A2C2010792, 2021R1A4A1032114, and NRF-2019R1A2C1088965). 


\section{Author contributions}

S.-H.S. and W.C. conceived the project. S.P. and Y.J. constructed the experimental setup. S.P. conducted superresolution imaging experiments with the help of M.K., S.K. and S.P.* With guidance of Y.J., J.H.H., S.-H.S. and W.C., S.P. performed data analysis. J.H.H., S.K.* and H.-C.P. prepared biological samples and provided guidance for data interpretation. S.P., S.-H.S. and W.C. prepared the manuscript and all authors contributed to finalizing the manuscript. S.-H.S. and W.C. supervised the project. S.P. and S.P.* indicate Sanghyeon Park and Sangjun Park, respectively. S.K. and S.K.* correspond to Sangyoon Ko and Suhyun Kim, respectively. 
bioRxiv preprint doi: https://doi.org/10.1101/2021.11.18.469175; this version posted November 20, 2021. The copyright holder for this preprint (which was not certified by peer review) is the author/funder. All rights reserved. No reuse allowed without permission.

\section{References}

1. Betzig, E. et al. Imaging Intracellular Fluorescent Proteins at Nanometer Resolution. Science 313, 1642-1645 (2006)

2. Rust, M. J., Bates, M. \& Zhuang, X. Sub-diffraction-limit imaging by stochastic optical reconstruction microscopy (STORM). Nat. Methods 3, 793-795 (2006).

3. Sharonov, A. \& Hochstrasser, R. M. Wide-field subdiffraction imaging by accumulated binding of diffusing probes. Proc. Natl. Acad. Sci. 103, 18911-18916 (2006).

4. Sigal, Y. M., Zhou, R. \& Zhuang, X. Visualizing and discovering cellular structures with super-resolution microscopy. Science 361, 880-887 (2018).

5. Kim, J. et al. Oblique-plane single-molecule localization microscopy for tissues and small intact animals. Nat. Methods 16, 853-857 (2019).

6. Chen, B. C. et al. Lattice light-sheet microscopy: Imaging molecules to embryos at high spatiotemporal resolution. Science 346, (2014).

7. Booth, M., Andrade, D., Burke, D., Patton, B. \& Zurauskas, M. Aberrations and adaptive optics in superresolution microscopy. Microscopy 64, 251-261 (2015).

8. Ji, N. Adaptive optical fluorescence microscopy. Nat. Methods 14, 374-380 (2017).

9. Tyson, R. K. Principles of Adaptive Optics. (CRC Press, 2015).

10. Booth, M. J. Adaptive optical microscopy: the ongoing quest for a perfect image. Light Sci. Appl. 3, e165e165 (2014).

11. Mlodzianoski, M. J. et al. Active PSF shaping and adaptive optics enable volumetric localization microscopy through brain sections. Nat. Methods 15, 583-586 (2018).

12. Burke, D., Patton, B., Huang, F., Bewersdorf, J. \& Booth, M. J. Adaptive optics correction of specimeninduced aberrations in single-molecule switching microscopy. Optica 2, 177 (2015).

13. Patton, B. R. et al. Three-dimensional STED microscopy of aberrating tissue using dual adaptive optics. Opt. Express 24, 8862 (2016).

14. Gould, T. J., Burke, D., Bewersdorf, J. \& Booth, M. J. Adaptive optics enables 3D STED microscopy in aberrating specimens. Opt. Express 20, 20998-21009 (2012).

15. Zheng, W. et al. Adaptive optics improves multiphoton super-resolution imaging. Nat. Methods 14, 869-872 (2017).

16. Izeddin, I. et al. PSF shaping using adaptive optics for three-dimensional single-molecule super-resolution imaging and tracking. Opt. Express 20, 4957 (2012).

17. Turcotte, R. et al. Dynamic super-resolution structured illumination imaging in the living brain. Proc. Natl. Acad. Sci. 116, 9586-9591 (2019).

18. Siemons, M. E., Hanemaaijer, N. A. K., Kole, M. H. P. \& Kapitein, L. C. Robust adaptive optics for localization microscopy deep in complex tissue. Nat. Commun. 12, 1-9 (2021).

19. Tehrani, K. F., Xu, J., Zhang, Y., Shen, P. \& Kner, P. Adaptive optics stochastic optical reconstruction microscopy (AO-STORM) using a genetic algorithm. Opt. Express 23, 13677 (2015).

20. Xu, F. et al. Three-dimensional nanoscopy of whole cells and tissues with in situ point spread function retrieval. Nat. Methods 17, 531-540 (2020).

21. Kang, S. et al. High-resolution adaptive optical imaging within thick scattering media using closed-loop accumulation of single scattering. Nat. Commun. 8, (2017).

22. Kim, M. et al. Label-free neuroimaging in vivo using synchronous angular scanning microscopy with singlescattering accumulation algorithm. Nat. Commun. 10, 1-9 (2019).

23. Yoon, S., Lee, H., Hong, J. H., Lim, Y.-S. \& Choi, W. Laser scanning reflection-matrix microscopy for aberration-free imaging through intact mouse skull. Nat. Commun. 11, 5721 (2020).

24. Ovesný, M., Kř́řžek, P., Borkovec, J., Švindrych, Z. \& Hagen, G. M. ThunderSTORM: A comprehensive ImageJ plug-in for PALM and STORM data analysis and super-resolution imaging. Bioinformatics 30, 23892390 (2014).

25. Pleiner, T., Bates, M. \& Görlich, D. A toolbox of anti-mouse and anti-rabbit IgG secondary nanobodies. $J$. Cell Biol. 217, 1143-1154 (2018).

26. Bates, M., Huang, B., Dempsey, G. T. \& Zhuang, X. Multicolor super-resolution imaging with photoswitchable fluorescent probes. Science 317, 1749-1753 (2007).

27. Endesfelder, U., Malkusch, S., Fricke, F. \& Heilemann, M. A simple method to estimate the average localization precision of a single-molecule localization microscopy experiment. Histochem. Cell Biol. 141, 629638 (2014).

28. Nieuwenhuizen, R. P. J. et al. Measuring image resolution in optical nanoscopy. Nat. Methods 10, 557-562 (2013). 
29. Münzel, E. J. et al. Claudin $\mathrm{k}$ is specifically expressed in cells that form myelin during development of the nervous system and regeneration of the optic nerve in adult zebrafish. Glia 60, 253-270 (2012).

30. Czopka, T., ffrench-Constant, C. \& Lyons, D. A. Individual oligodendrocytes have only a few hours in which to generate new myelin sheaths invivo. Dev. Cell 25, 599-609 (2013).

31. Almeida, R. G., Czopka, T., Ffrench-Constant, C. \& Lyons, D. A. Individual axons regulate the myelinating potential of single oligodendrocytes in vivo. Development 138, 4443-4450 (2011).

32. Preston, M. A. \& Macklin, W. B. Zebrafish as a model to investigate CNS myelination. Glia 63, 177-193 (2015).

33. Snaidero, N. et al. Myelin membrane wrapping of CNS axons by PI(3,4,5)P3-dependent polarized growth at the inner tongue. Cell 156, 277-290 (2014).

34. Sobottka, B., Ziegler, U., Kaech, A., Becher, B. \& Goebels, N. CNS live imaging reveals a new mechanism of myelination: The liquid croissant model. Glia 59, 1841-1849 (2011).

35. Dempsey, G. T., Vaughan, J. C., Chen, K. H., Bates, M. \& Zhuang, X. Evaluation of fluorophores for optimal performance in localization-based super-resolution imaging. Nat. Methods 8, 1027-1036 (2011).

36. Sigal, Y. M., Speer, C. M., Babcock, H. P. \& Zhuang, X. Mapping Synaptic Input Fields of Neurons with Super-Resolution Imaging. Cell 163, 493-505 (2015).

37. Dani, A., Huang, B., Bergan, J., Dulac, C. \& Zhuang, X. Superresolution Imaging of Chemical Synapses in the Brain. Neuron 68, 843-856 (2010).

38. Xu, J. et al. Super-resolution imaging reveals the evolution of higher-order chromatin folding in early carcinogenesis. Nat. Commun. 11, 1899 (2020).

39. Schindelin, J. et al. Fiji: An open-source platform for biological-image analysis. Nat. Methods 9, 676-682 (2012). 


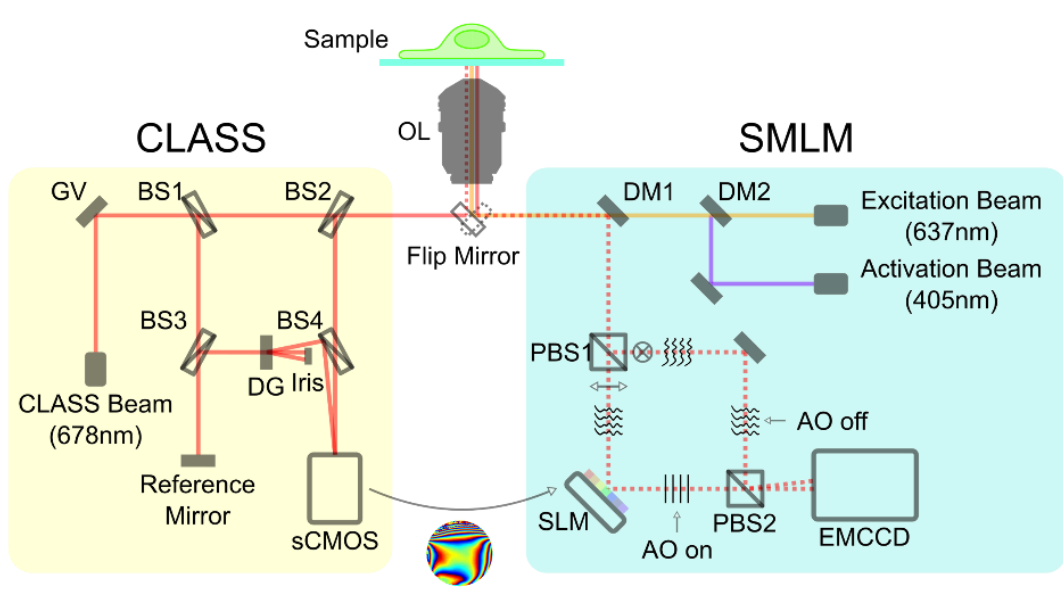

b $\quad \mathrm{AO}$ off

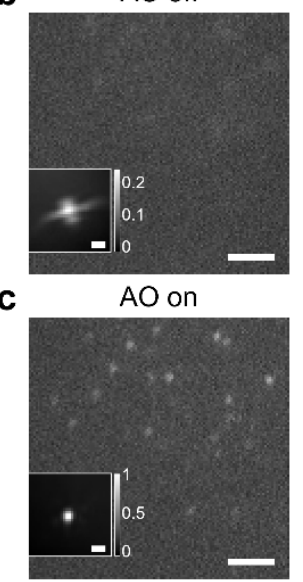

Aberration Map

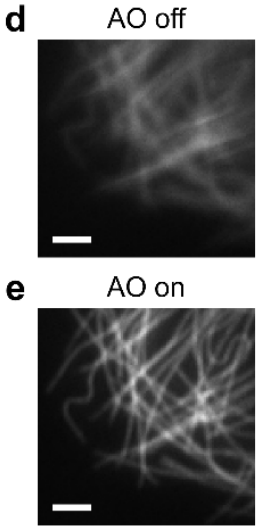

h

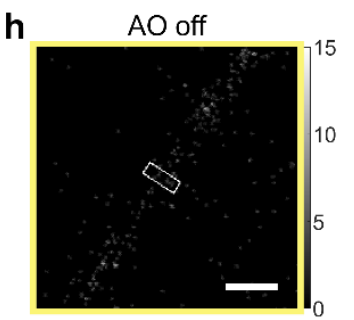

I

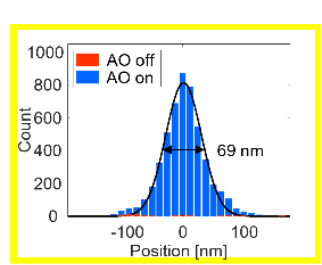

f

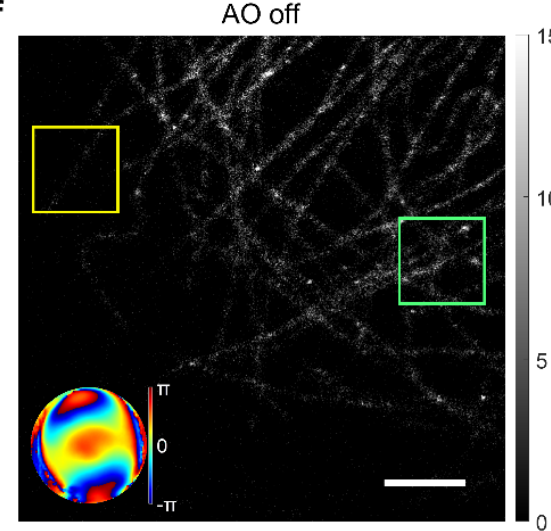

i $\quad$ AO on

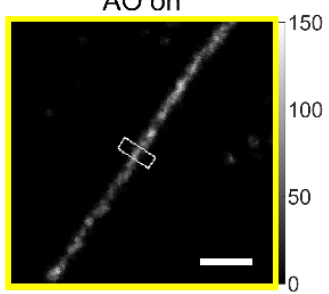

m

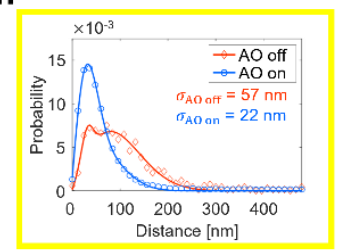

n g

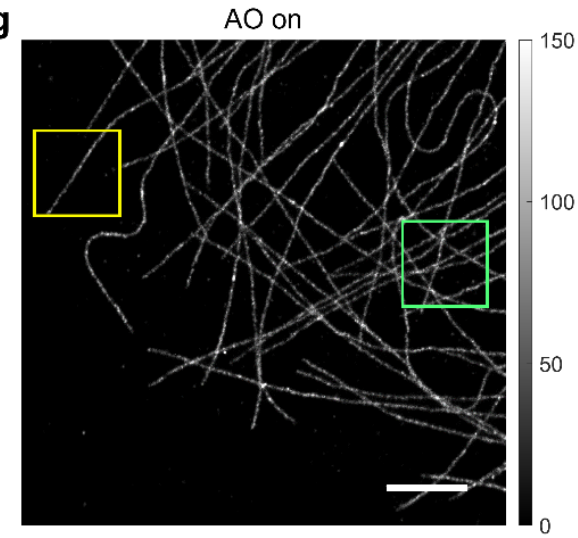

$\mathrm{AO}$ off
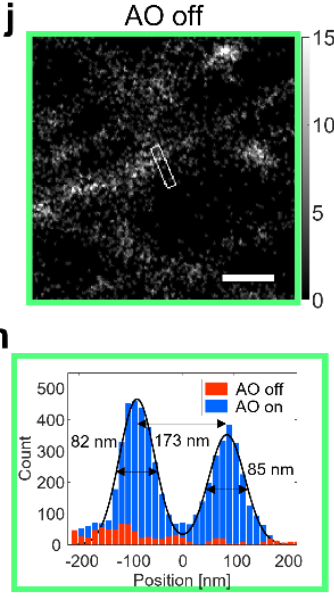

$\mathbf{k} \quad \mathrm{AO}$ on
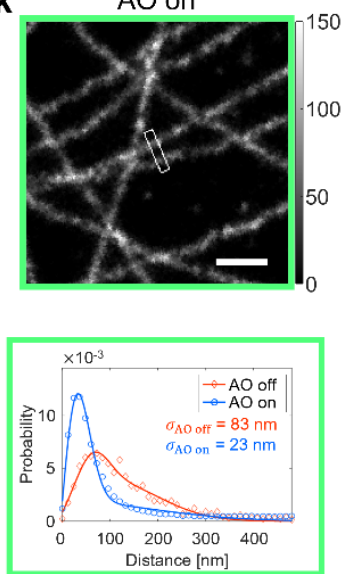

Figure 1. Experimental layout and demonstration of restoring distorted single-molecule PSFs in a COS-7 cell with an aberration layer. a, Scheme of experimental setup comprising CLASS microscope (yellow box) and SML microscope (cyan box). GV: two-axis galvanometer mirror, DG: diffraction grating, BS1-4: beam splitters, DM1-2: dichroic mirrors, PBS1-2: polarising beam splitters, SLM: spatial light modulator, and grey rectangles without labels: mirrors. b-c, Single-frame raw images of aberrated cellular microtubules simultaneously recorded without and with AO, respectively. Images are normalized with respect to AO on. Insets, ensemble-averaged PSFs obtained from first 10,000 frames with normalization with respect to AO on. d-e, Diffraction-limited fluorescence images without and with AO, respectively. Images are normalized with respect to AO on. f-g, SMLM images without and with $\mathrm{AO}$, respectively. Inset in $\mathbf{f}$, aberration correction map whose radius is $1.2 / \lambda$ in spatial frequency. $\mathbf{h}-\mathbf{k}$, Magnified views of yellow boxes $(\mathbf{h}-\mathbf{i})$ and green boxes $(\mathbf{j}-\mathbf{k})$ in $\mathbf{f}-\mathbf{g}$, respectively. $\mathbf{l}$, Cross-sectional profiles of white boxes in $\mathbf{h}-\mathbf{i}$. $\mathbf{m}$, Nearest neighbor analysis results for yellow boxes and other areas where microtubules were isolated. $\mathbf{n}$, Cross-sectional profiles of white boxes in $\mathbf{j}-\mathbf{k}$. $\mathbf{o}$, Same as $\mathbf{m}$, but for the green boxes and other 
areas where microtubules were confluent. Color bars in $\mathbf{f}-\mathbf{g}$ and $\mathbf{h}-\mathbf{k}$ show the localization numbers. Scale bars, $2.5 \mu \mathrm{m}(\mathbf{b}-\mathbf{g})$, and $500 \mathrm{~nm}$ (insets in b-d and h-k).
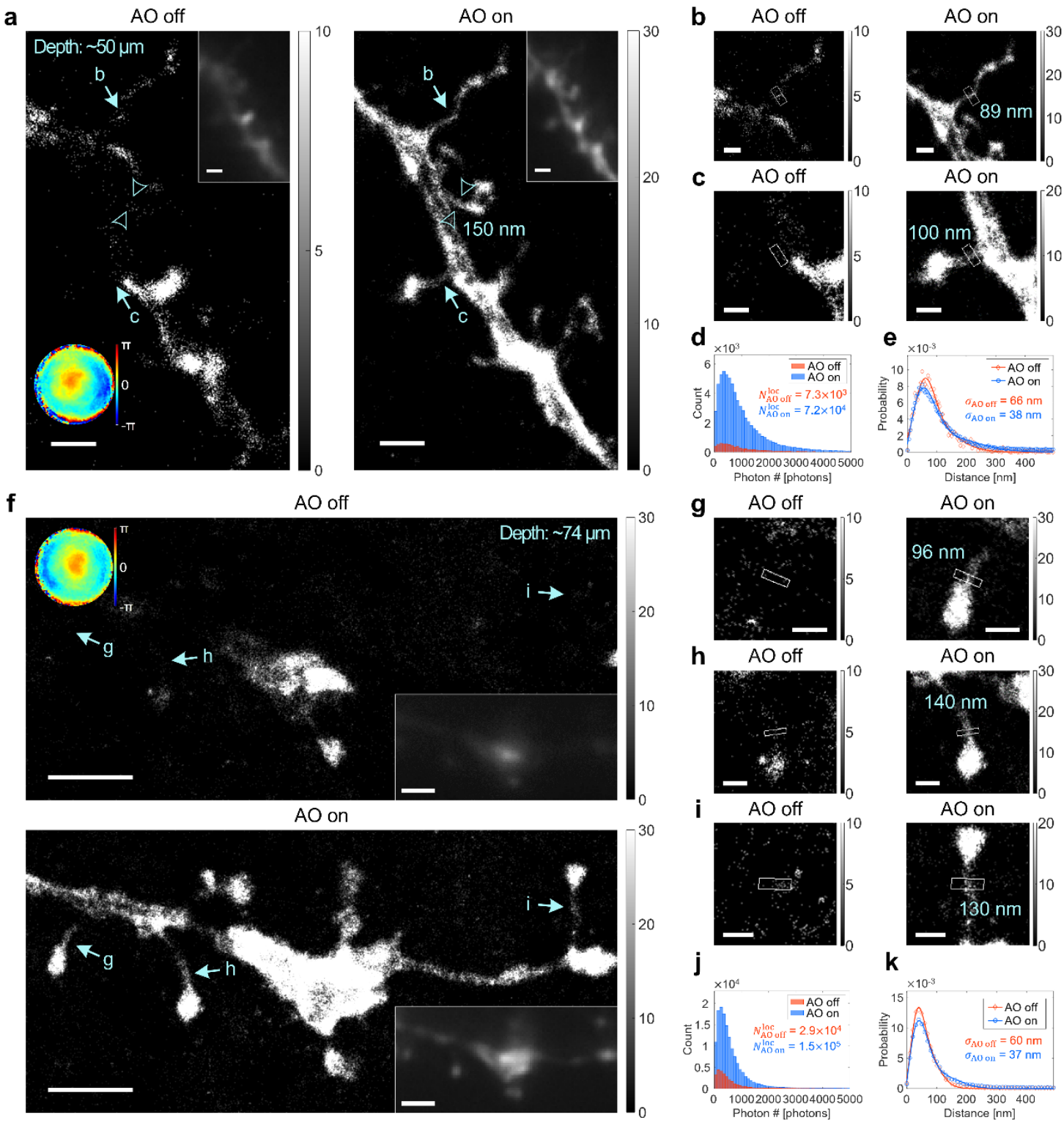

Figure 2. Deep-tissue SMLM images of dendritic spines in mouse brain slices without and with AO. a, SMLM images of dendritic spines at $50 \mu \mathrm{m}$ depth in an approximately 150 - $\mu \mathrm{m}$-thick mouse brain slice without and with AO. Top-right insets show diffraction-limited fluorescence images of each SMLM image. Bottom-left inset in AO-off image shows aberration correction map. Color bar presents phase in radians. b-c, Magnified views of regions indicated by solid arrows in a. FWHM values of dendritic spine necks (white boxes) are indicated in AO-on images. d-e, Histogram of photon numbers per emission PSF (d) and nearest neighbor analysis results (e) of a. f, SMLM images of dendritic spines at $74 \mu \mathrm{m}$ depth in an approximately $200 \mu \mathrm{m}$ thick mouse brain slice without and with AO. Bottom-right insets show diffraction-limited fluorescence image of each SMLM image. Top-left inset in AO-off image shows aberration correction map. Color bar presents phase in radians. g-i, Magnified views of regions indicated by solid arrows in f. FWHM values of dendritic spine necks (white boxes) are indicated in AO-on images. j-k, Histogram of photon number per emission PSF (j) and nearest neighbor analysis results (k) of $\mathbf{f}$. Color bars in $\mathbf{a}-\mathbf{c}$ and $\mathbf{f}-\mathbf{i}$ show localization numbers. Scale bars, $2 \mu \mathrm{m}$ (a, and insets in $\mathbf{a})$, $500 \mathrm{~nm}(\mathbf{b}-\mathbf{c}), 2.5 \mu \mathrm{m}$ (f and insets in $\mathbf{f})$, and $500 \mathrm{~nm}(\mathbf{g}-\mathbf{i})$. 


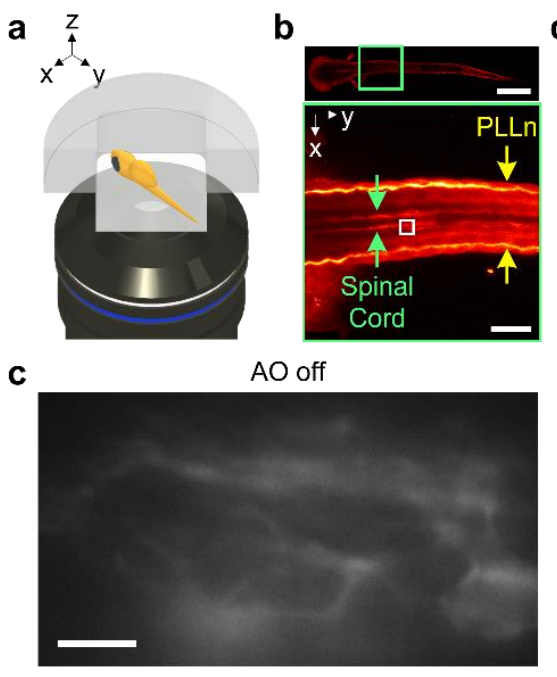

AO on
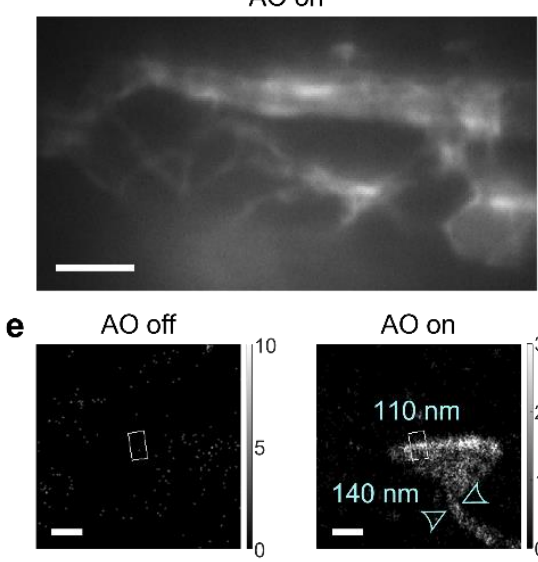

g

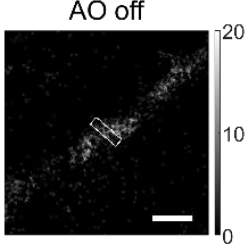

d

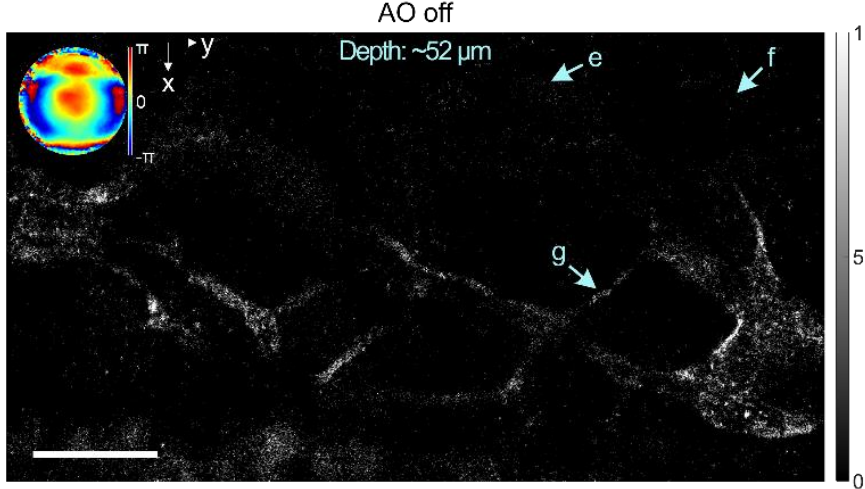

$\mathrm{AO}$ on

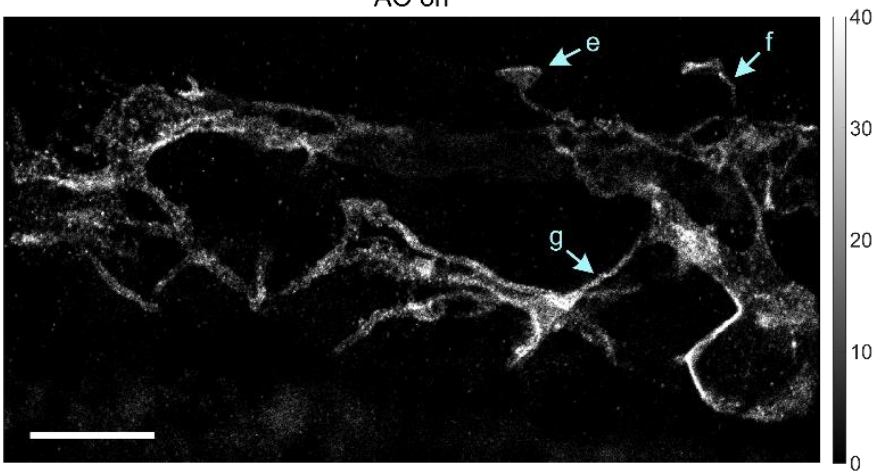

f

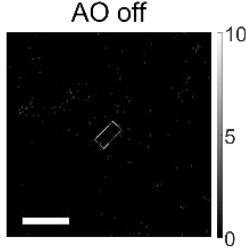

h
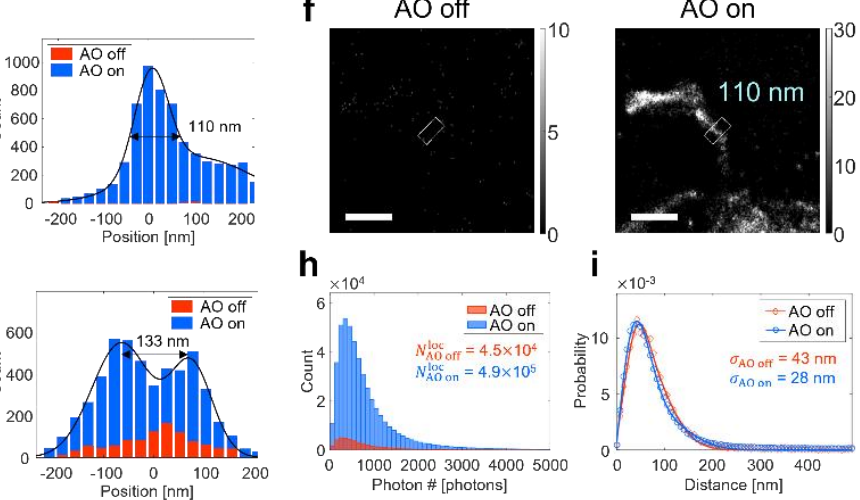

Figure 3. Deep-tissue SMLM images of an intact zebrafish without and with AO. a, Schematic layout of a zebrafish sample on its back. It should be noted that the zebrafish size is exaggerated. $\mathbf{b}$, Confocal fluorescence images of zebrafish expressing GFP in oligodendrocytes. Entire view (top, b) and magnified view (bottom, b) of green box (top, b). White box in (bottom, b) shows view field of SML microscope. PLLn: peripheral lateral line nerve. c, Diffraction-limited fluorescence images of the central nervous system and oligodendrocytes at $52 \mu \mathrm{m}$ depth in a fixed 3.5-dpf intact zebrafish without and with AO. d, SMLM images of same view fields in $\mathbf{c}$ without and with AO. Inset shows aberration correction map. e-g, Magnified views of regions indicated by arrows in $\mathbf{d}$. Cross-sectional profiles of white boxes in magnified views are shown in the rightmost columns in e-g. h-i, Histogram of photon number per emission PSF (h) and nearest neighbor analysis results (i) of d. Scale bars: 500 $\mu \mathrm{m}$ (top, b), $100 \mu \mathrm{m}$ (bottom, b), $5 \mu \mathrm{m}$ (c-d), $500 \mathrm{~nm}$ (e-g), $1 \mu \mathrm{m}$ (f). 
a

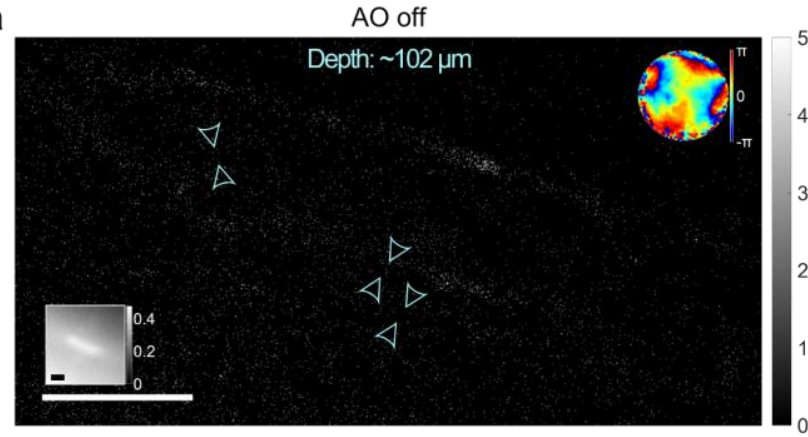

b

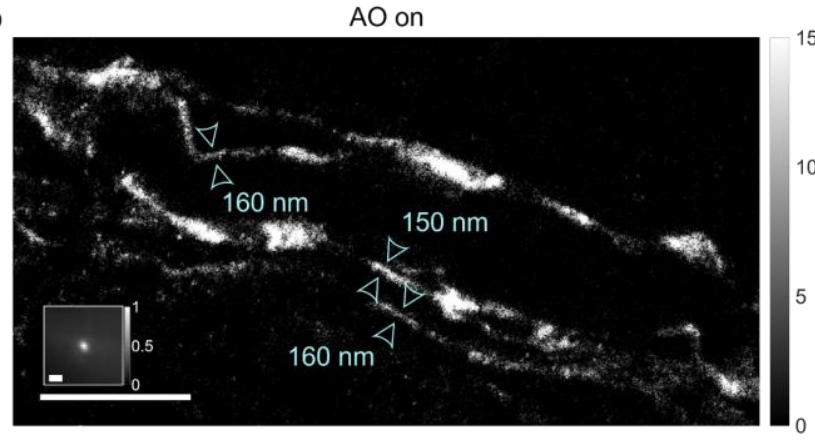

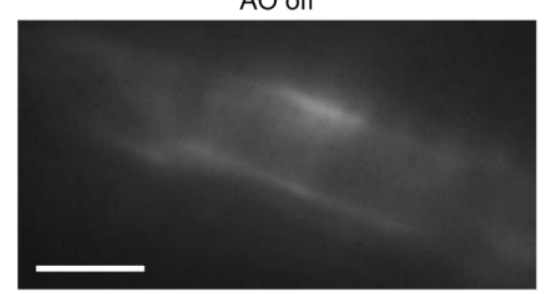

d

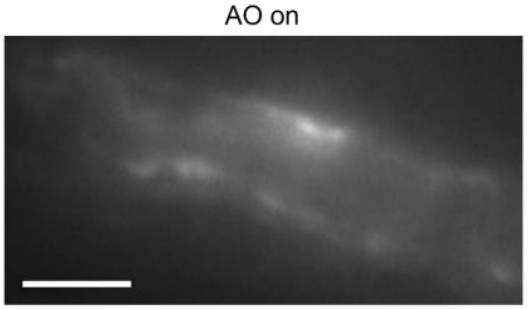

e

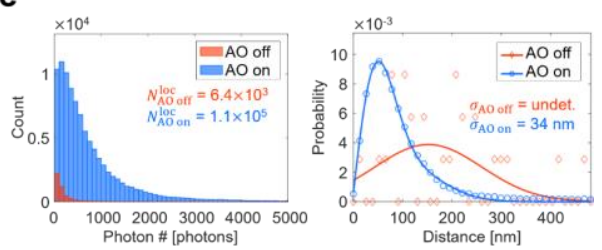

Figure 4. Over-100- $\mu \mathrm{m}$-depth deep-tissue SMLM images of an intact zebrafish. a and $\mathbf{b}$, SMLM images of oligodendrocytes wrapping the spinal cord at $102 \mu \mathrm{m}$ depth in a 5-dpf zebrafish without (a) and with (b) AO, respectively. Numbers in nm indicate FWHM values of tubular structures indicated by arrowheads. Top-right inset shows aberration correction map. Bottom-left insets present ensemble-averaged PSFs in the first 10,000 frames with normalization with respect to AO on. c-d, Diffraction-limited fluorescence images of $\mathbf{a}-\mathbf{b}$, respectively. e, Histogram of photon number per emission PSF (left) and nearest neighbor analysis results (right). It should be noted that $\sigma_{\mathrm{AO} \text { off }}$ could not be determined because the localization number was too low. Color bars in a-b present localization numbers. Scale bars: $5 \mu \mathrm{m}$ (a-d) and $500 \mathrm{~nm}$ (bottom-left insets in a-b). 